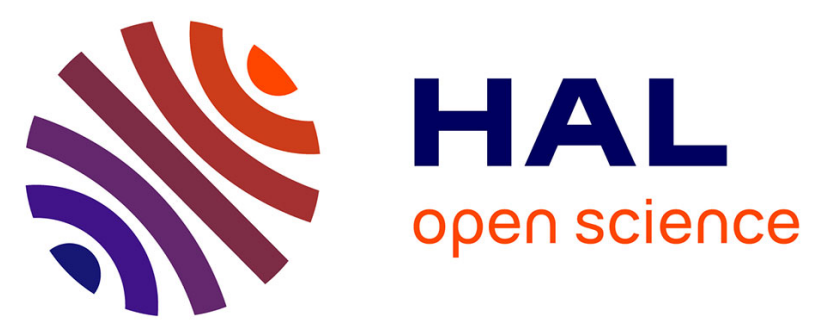

\title{
Performance Analysis of REM-Based Handover Algorithm for Multi-Tier Cellular Networks
}

Cristo Suarez-Rodriguez, B A Jayawickrama, Ying He, Faouzi Bader, Michael Heimlich

\section{- To cite this version:}

Cristo Suarez-Rodriguez, B A Jayawickrama, Ying He, Faouzi Bader, Michael Heimlich. Performance Analysis of REM-Based Handover Algorithm for Multi-Tier Cellular Networks. The 28th Annual IEEE International Symposium on Personal, Indoor and Mobile Radio Communications (IEEE PIMRC 2017), Oct 2017, Montréal, Canada. 10.1109/pimrc.2017.8292488 . hal-01705734

\section{HAL Id: hal-01705734 \\ https://hal.science/hal-01705734}

Submitted on 9 Feb 2018

HAL is a multi-disciplinary open access archive for the deposit and dissemination of scientific research documents, whether they are published or not. The documents may come from teaching and research institutions in France or abroad, or from public or private research centers.
L'archive ouverte pluridisciplinaire HAL, est destinée au dépôt et à la diffusion de documents scientifiques de niveau recherche, publiés ou non, émanant des établissements d'enseignement et de recherche français ou étrangers, des laboratoires publics ou privés. 


\title{
Performance Analysis of REM-Based Handover Algorithm for Multi-Tier Cellular Networks
}

\author{
Cristo Suarez-Rodriguez ${ }^{* \ddagger}$, B. A. Jayawickrama ${ }^{\dagger}$, Ying $\mathrm{He}^{\dagger}$, Faouzi Bader ${ }^{\ddagger}$ and Michael Heimlich*† \\ *Department of Engineering, Macquarie University, Sydney, Australia \\ Email: cristo.suarez-rodriguez@hdr.mq.edu.au, michael.heimlich@mq.edu.au \\ †University of Technology Sydney, Global Big Data Technologies Centre, Sydney, Australia \\ Email: \{beeshanga.jayawickrama, ying.he\}@uts.edu.au \\ ${ }^{\ddagger}$ SCEE/IETR, CentraleSupélec, Rennes, France \\ Email: faouzi.bader@supelec.fr
}

\begin{abstract}
The advent of $5 \mathrm{G}$ networks, where a plethora of spectrum-sharing schemes are expected to be adopted as an answer to the ever-growing users' need for data traffic, will require addressing mobility ubiquitously. The trend initiated with the deployment of heterogeneous networks and past standards will give way to a multitiered network where different services will coexist, such as device-to-device, vehicle-to-vehicle or massive-machine communications. Because of the high variability in the cell sizes given the different transmit powers, the classical handover process, which relies solely on measurements, will lead to an unbearable network overhead as a consequence of the high number of handovers. The use of spatial databases, also known as radio environment maps (REM), was first introduced as a tool to detect opportunistic spectrum access opportunities in cognitive radio applications. Since then, REM usage has been widely expanded to cover deployment optimization, interference management or resource allocation to name a few. In this paper, we introduce a handover algorithm that can predict the best network connection for the current user's trajectory from a radio environment map. We consider a geometric approach to derive the handover and handover-failure regions and compare the current handover algorithm used in Long-Term Evolution with our proposed one. Results show a drastic reduction in the number of handovers while maintaining a trade-off between the ping-pong handover and the handover-failure probabilities.
\end{abstract}

Index Terms-Radio Environment Maps, Handover, Heterogeneous Networks.

\section{INTRODUCTION}

Current mobile communications are struggling to service data-hungry users as reported by Cisco in their yearly authoritative forecast [1]. Among the envisioned trends, it is expected that global mobile traffic will increase sevenfold for the covered period $(2016$ - 2021) continuing a steady eighteenfold escalation over the last five years. Approximately $60 \%$ of all mobile data traffic is being offloaded onto the fixed network through operator-owned Wi-Fi hotspots or small cells (micro-
, nano-, pico-, and femtocells), which represents an accumulated monthly traffic of 10.7 exabytes. So as to fulfill users' demands ubiquitously, mobility across different technologies operating in several frequency bands will be required.

The inclusion of small cells in the Long-TermEvolution (LTE) family of standards underlying the coverage of macrocells to improve the capacity per unit area was supposed to be the first step in this direction. However, it also brought a series of challenges related to the handover (HO) process [2]. While in homogeneous networks (macro only) user equipments (UE) only cope with one tier of base stations (BS), which translates into a similar set of transmit powers and handover-related parameters such as the time-to-trigger (TTT) and the handover hysteresis margin (HMM), in heterogeneous networks (HetNet) the existence of multiple tiers invalidates the classic approach [3]. Not only does the presence of more than one tier affects the experienced quality of service (QoS) from the users' point of view, but so also does their velocity. For example, high-speed users tend to experience frequent HOs to/from the same pair of BSs within a short time frame, e.g. $1 \mathrm{~s}$, which is the so-called ping-pong effect. Similarly, they reach inner regions of the small cells' coverage area before performing a $\mathrm{HO}$, therefore the degradation in the wideband signal-to-interferenceplus-noise ratio (SINR) might cause a HO failure.

Next-generation cellular networks, LTE Advanced Pro (LTE-A Pro) and 5G New Radio (NR) as branded by the $3^{\text {rd }}$ Generation Partnership Project (3GPP), take this a step further and include spectrum-sharing paradigms, specially below $6 \mathrm{GHz}$, in both licensed (vertical sharing) and unlicensed bands (horizontal sharing), unlocking new use cases [4]. In the former, Licensed Shared Access (LSA) in Europe and Citizen Broadband Radio Service (CBRS) in the US divide 
users into tiers (tiered sharing) between incumbents (owners) and licensees (with several degrees of privileges in the case of CBRS). In the latter, from Release 13, LTE-Unlicensed (LTE-U) - first in the US, Korea, and India - and Licensed-Assisted Access (LAA) available worldwide - allow mobile network operators (MNO) to employ LTE in unlicensed spectrum with the objective of adding more available capacity by means of carrier aggregation while ensuring co-existence with Wi-Fi (spectrum aggregation). At the same time, MNOs can reuse existing Wi-Fi deployments through LTE Wi-Fi Link Aggregation (LWA) with the same purpose (technology aggregation). This makes it necessary to revisit the $\mathrm{HO}$ process one more time.

A comprehensive survey of handover decision algorithms for femtocells in LTE-A is delivered in [5], where the authors classify state-of-the-art algorithms according to the $\mathrm{HO}$ decision criterion using: a) received signal strength (RSS), b) speed, c) cost function, d) interference awareness and e) energy efficiency. Yet they do not cover context awareness as in [6], which shows a Markov-based framework that exploits some scenario parameters (the path-loss coefficients, the UE speed, and the cell load factors among others) to derive an optimal context-dependent TTT. Similarly, in [7] a Markov-based mobility prediction method is derived from diurnal mobility patterns (from home to work, then to a supermarket, etc.) to predict the next cell and avoid coverage holes (tunnels) by buffering data beforehand. In [8], for instance, the authors estimate the distance that a user traverses based on RSS measurements and calculate distance thresholds according to the probability of handover failure and unnecessary handover.

In this work, we are interested in using predicted incomplete channel states from radio environment maps (REM) to reduce the number of unnecessary handovers, i.e. ping-pong handovers, since they incur a burden in the form of resource consumption and network overhead. A REM stores the spatial distribution of the average RSS, therefore the effect of path loss and shadowing is captured while fast-fading is averaged out. We present a novel handover algorithm that makes use of REMs and evaluate its performance in a reference scenario. Indeed, because the proposed algorithm scheme relies on the same event-triggered mechanism as does LTE-A, they can be easily compared to each other. However, in our case, we need UEs to have the capability of positioning using either a Global Navigation Satellite System or an LTE-A networkassisted technique along with the measurement reports, as already supported by the Minimization of Drive Test (MDT) in LTE-A as in [9], where the authors introduce the concept of a forecast scheduler for users with high mobility employing SINR values provided by a REM. Moreover, we assume that REMs are stored and managed by the mobile network operator in a similar fashion to [10].

Section II presents an overview of the system model employed. Section III explains the REM-based handover algorithm. Section IV presents a performance comparison with the LTE-HO algorithm. Finally, Section $\mathrm{V}$ states the main findings of this work and a summary.

\section{SySTEM MODEL}

Consider two Base Stations (BS) operating in the same frequency band $f, s$ (serving) and $t$ (target), whose localizations and powers are $\left\{x_{s, t}, y_{s, t}\right\}$ and $P t_{s, t}$ respectively. The simplified path-loss model as a function of distance is given by [11]

$$
P r=P t+K-10 \gamma \log _{10}\left(\frac{d}{d_{0}}\right)
$$

where $\operatorname{Pr}$ is the received power at distance $d, P t$ is the transmit power, $K$ is the attenuation at distance $d_{0}$ and $\gamma$ is the path-loss exponent, which takes into account the unique characteristics of the propagation environment such as frequency, antenna height, etc. Without loss of generality, we assume that $d_{0}=1$ m. Then $K=20 \log _{10}(c / 4 / \pi / f)$, where $c$ represents the speed of light. Furthermore, let's consider a UE in location $\{x, y\}$ originally camped on $s$ who is moving towards $t$ with a linear uniform motion and speed $v$. We assume that this user is following the LTE-HO policy, which can be summarized as

$$
P r_{s}<P r_{t}-\alpha
$$

where $P r_{s, t}$ are each BS's received power at the user's location according to (1) and $\alpha$ is the Hysteresis Handover Margin (HHM) used to prevent unnecessary handovers. Plugging (1) into (2) we obtain

$$
\begin{gathered}
P t_{s}+K_{s}-10 \gamma \log _{10}\left(\sqrt{\left(x-x_{s}\right)^{2}+\left(y-y_{s}\right)^{2}}\right)< \\
P t_{t}+K_{t}-10 \gamma \log _{10}\left(\sqrt{\left(x-x_{t}\right)^{2}+\left(y-y_{t}\right)^{2}}\right)-\alpha .
\end{gathered}
$$

In the considered system model we can drop $K_{s, t}$ since both BSs operate in the same band, otherwise we could define the constant $K=K_{s}-K_{t}$ and carry on with it through the calculations. After some rearrangement of (3), we have

$$
\begin{gathered}
(1-\mathcal{Z}) x^{2}+(1-\mathcal{Z}) y^{2}+2\left(\mathcal{Z} x_{t}-x_{s}\right) x+ \\
2\left(\mathcal{Z} y_{t}-y_{s}\right) y+x_{s}^{2}-\mathcal{Z} x_{t}^{2}+y_{s}^{2}-\mathcal{Z} y_{t}^{2}>0
\end{gathered}
$$

where $\mathcal{Z}$ is given by

$$
\mathcal{Z}=10^{\frac{\alpha+P t_{s}-P t_{t}}{5 \gamma}} .
$$


Note that (4) is a quadratic inequality in two variables which describes a conic section of the form $A x^{2}+C y^{2}+D x+E y+F>0$, which divides the Cartesian space into two: handover and non-handover regions. The discriminant $-4 A C=-4(1-\mathcal{Z})^{2}$ tells what kind of region (4) is. Depending on the value of $\mathcal{Z} \in[0, \infty)$ we can have:

1) $\forall \mathcal{Z}-\{1\}$, the equation represents an ellipse.

- If $A=C=(1-\mathcal{Z})$, which is the case, the equation represents a circle (a degenerate ellipse).

2) $\mathcal{Z}=1 \rightarrow A=C=0$, the equation represents a straight line.

In the first case, the circle is centered at $c_{h}=$ $\left\{x_{h}, y_{h}\right\}$ with squared radius $r_{h}^{2}$ whose expressions are

$$
\begin{aligned}
x_{h} & =-\frac{\mathcal{Z} x_{t}-x_{s}}{1-\mathcal{Z}} \\
y_{h} & =-\frac{\mathcal{Z} y_{t}-y_{s}}{1-\mathcal{Z}} \\
r_{h}^{2} & =\frac{\mathcal{Z}}{(1-\mathcal{Z})^{2}}\left[\left(x_{t}-x_{s}\right)^{2}+\left(y_{t}-y_{s}\right)^{2}\right] .
\end{aligned}
$$

In the second case, the straight line has a slope $m, x_{\text {intercept }}$ (if $D$ is nonzero) and $y_{\text {intercept }}$ (if $E$ is nonzero). In this case, the UE will hand over to the closest BS since the region does not form a closed polygon. Note that the transmit powers do not need to be the same to obtain a straight line (indeed $\left.\alpha+P t_{s}-P t_{t}=0\right)$.

$$
\begin{aligned}
m & =-\frac{\mathcal{Z} x_{t}-x_{s}}{\mathcal{Z} y_{t}-y_{s}} \\
x_{\text {intercept }} & =-\frac{x_{s}^{2}-x_{t}^{2}+y_{s}^{2}-y_{t}^{2}}{2\left(x_{t}-x_{s}\right)} \\
y_{\text {intercept }} & =-\frac{x_{s}^{2}-x_{t}^{2}+y_{s}^{2}-y_{t}^{2}}{2\left(y_{t}-y_{s}\right)} .
\end{aligned}
$$

There is one more general scenario where the pathloss exponent $\gamma$ is different between the BSs, as normally occurs in HetNets. In such a case, the analysis requires an approximation like a Taylor expansion series which leads to an ellipse. Despite this, under the assumption that one of the cells has a transmit power significantly lower than the other, it converges to a circle as in [12]. Our following analysis will hold as long as the circle center and radius are known.

Similarly, we can derive handover failure regions for getting out-of-sync for the serving cell and the target cell. As defined in [2], the wideband SINR threshold to declare a handover failure is $Q_{\text {out }}=$ $8 \mathrm{~dB}$. Consequently, substituting $\alpha$ in (2) for $Q_{\text {out }}$ $\left(P r_{s}<P r_{t}+Q_{\text {out }}\right)$, we can obtain $c_{i}=\left\{x_{i}, y_{i}\right\}$ and $r_{i}$ for the inbound handover-failure (HF) region. The outbound HF region, whose parameters are $c_{o}=\left\{x_{o}, y_{o}\right\}$

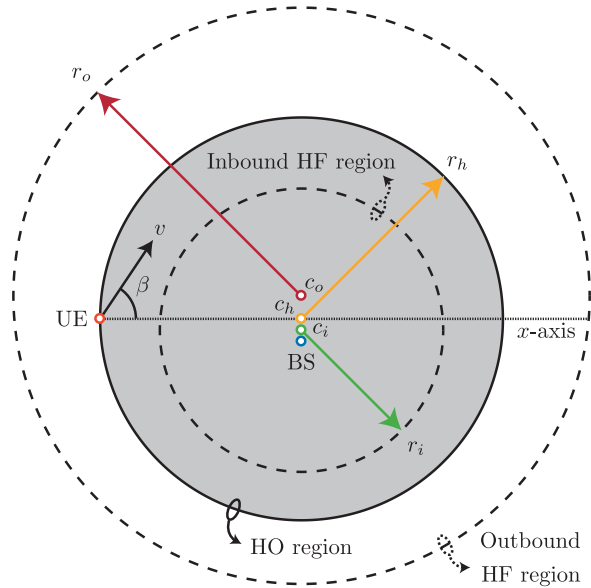

Fig. 1. Reference scenario: target cell (BS) and different regions (HO, inbound HF and outbound HF) with their respective centers and radii. The user (UE) is following a linear motion with speed $v$ and trajectory $\beta$.

and $r_{o}$, can be easily determined by exchanging the received powers. We consider that the UE is located right on the border between regions and starts moving from $s$ (serving) to $t$ (target) at speed $v$ with direction $\beta \in[-\pi / 2, \pi / 2]$, which is the angle with respect to the $x$-axis as shown in Fig. 1. As soon as the user enters the region already described, (2) must be met for at least a TTT of duration $T$ in order to perform a successful handover. A longer TTT reduces the probability of ping-pong handovers at the expense of increasing the probability of handover failures. Ping-pong handovers happen when performing two successful consecutive handovers between the same two cells within a minimum time of stay (ToS) of duration $T_{\mathrm{PP}}$. As a result, they reduce the efficiency of the network because they introduce a fair amount of overhead, so it is crucial to reduce their numbers while keeping a satisfactory QoS for the users. Note that the circles are not concentric, therefore the entry point does affect the geometry of the scenario as well as the results obtained. In the remainder of this paper, both cells will be vertically aligned.

\section{REM HANDOVER Algorithm Description}

Once the TTT is expired, our proposed algorithm (see Algorithm 1) predicts the user's position in a timespan $\Delta t$, assuming a simple uniform linear motion with speed $v$. The endpoint is then introduced in the corresponding REMs (each BS has one) to get an expected RSS value for a given cell. Despite the fact that we could just pick the strongest predicted cell in $\Delta t$, it is preferable to obtain a more unbiased measurement like the SINR because the expected QoS of the user will depend on the capacity of the channel rather than just the greatest received power. Nonetheless, since our system model consists of only two base stations and 


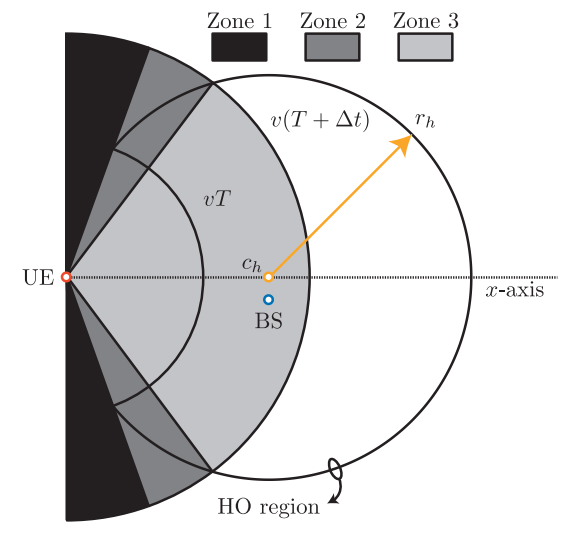

Fig. 2. A graphical description of the proposed REM handover algorithm.

we are just dealing with path loss for simplicity, our REMs can be simplified to the regions described in the previous section with $\alpha=0 \mathrm{~dB}$ (when the two received powers become equal). If the predicted user's position is within the handover region, our policy lets that user hand over to the new cell, otherwise, the user will remain connected to the source cell. An example of this predicted position is depicted in Fig. 2 where we can define the following zones:

- Zone 1: the user exits the handover region before the TTT expires, thus the $\mathrm{HO}$ algorithm is not triggered.

- Zone 2: the TTT expires, however the prediction lies outside the handover region resulting in an avoided HO.

- Zone 3: the TTT expires and the prediction is within the handover region, therefore the $\mathrm{HO}$ is allowed.

Taking all the previous parameters into account, our proposed policy to determine the strongest cell for handover can be expressed as

$$
P r_{t}>\left.P r_{s}\right|_{t+\Delta t} \quad \text { iff } \quad P r_{s}<P r_{t}-\left.\alpha\right|_{t}
$$

where all parameters from (2) remain. What this policy accomplishes is that a user will hand over to the strongest cell only if that same cell will remain the strongest one in the near future according to the user's trajectory. The different steps of the proposed algorithm are shown in Algorithm 1.

The procedure of the UE REM-HO takes place at the UE and it is identical to what is currently used in the LTE-A standard. Similarly, the second procedure labeled BS REM-HO is run on the operator's side.

\section{NumericAl RESUlts}

In this section, we show the simulated results for the probabilities of not handing over, handover failure and

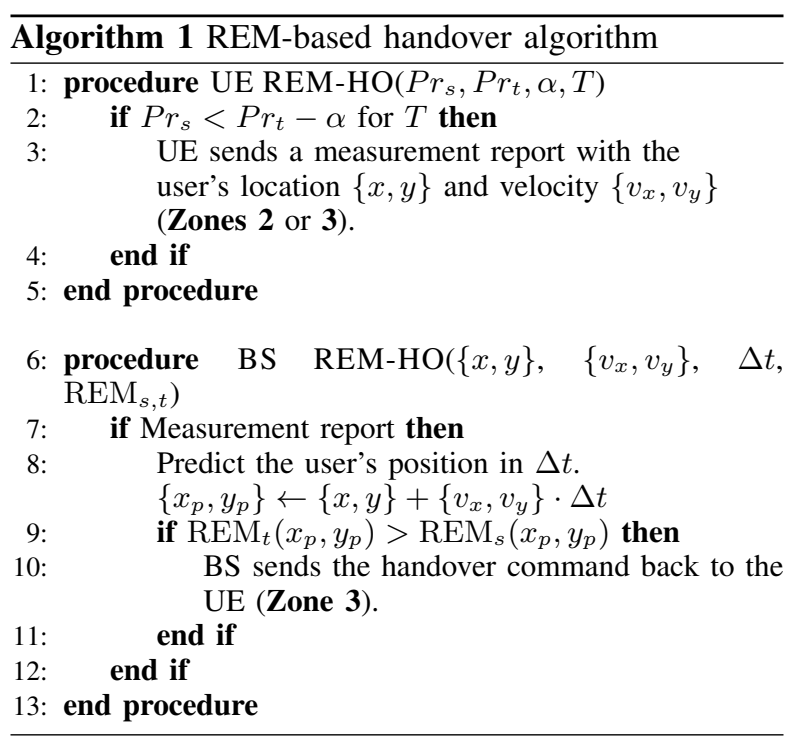

TABLE I

SIMULATION PARAMETERS

\begin{tabular}{l|l}
\hline Item & Value \\
\hline \hline$f$ & $2 \mathrm{GHz}$ \\
$\gamma$ & 2 \\
$\left\{x_{s}, y_{s}\right\}$ & $\{0,0\} \mathrm{m}$ \\
$P t_{s}$ & $49 \mathrm{dBm}$ \\
$\left\{x_{t}, y_{t}\right\}$ & $\{0,200\} \mathrm{m}$ \\
$P t_{t}$ & $30 \mathrm{dBm}$ \\
$\alpha$ & $0 \mathrm{~dB}$ \\
$Q_{\text {out }}$ & $-8 \mathrm{~dB}$ \\
$v$ & $\{0,10,20, \ldots, 100,110,120\} \mathrm{km} / \mathrm{h}$ \\
$\beta$ & {$[-\pi / 2, \pi / 2]$} \\
$T$ & $\{40,80,160,480\} \mathrm{ms}$ \\
$\Delta t$ & $2.5 \mathrm{~s}$ \\
$T_{\mathrm{PP}}$ & $1 \mathrm{~s}$ \\
\hline
\end{tabular}

ping-pong handover as well as a comparison with the LTE-HO algorithm. The simulations have been carried out in MATLAB and the main parameters can be seen in Table I. We assume omnidirectional antennas in both base stations and for the user and, as in the whole paper, only path loss is considered. Furthermore, the measurement period of LTE-A is ignored, so we have perfect knowledge of the received power at any moment. Finally, perfect location is also assumed.

In the first place, we want to evaluate the performance of both algorithms under different TTTs. In Fig. 3 (a), the probability of not handing over $\left(\mathbb{P}_{\mathrm{NHO}}\right)$ increases as expected with the TTT and the speed because the distance $v T$ is longer, thus there are more chances to abandon the handover region before the TTT expires. However, for our proposed algorithm the probability of not handing over reaches a maximum value of $74.23 \%$ for all speeds above $70 \mathrm{~km} / \mathrm{h}$, which is equivalent to the proportion of angles that do not 


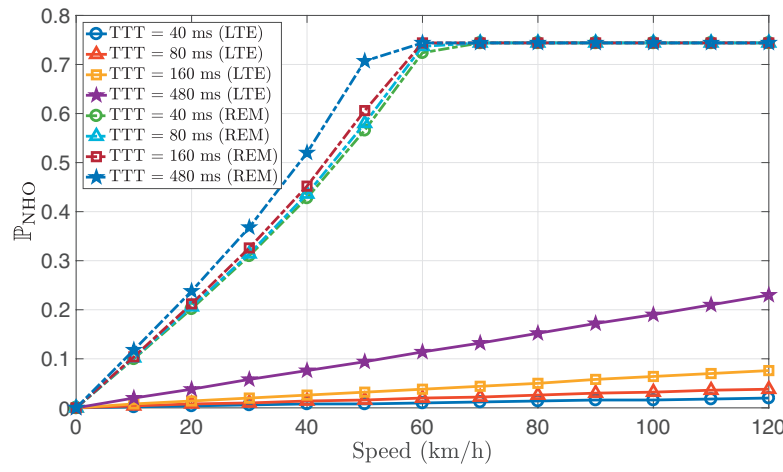

(a) Probability of not handing over.

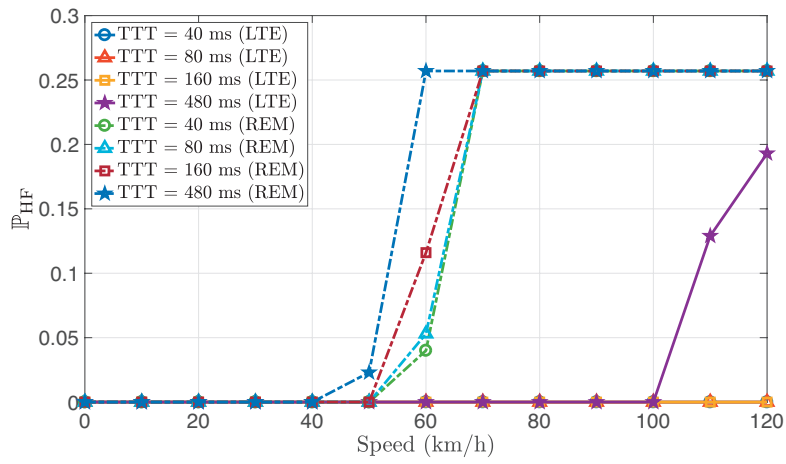

(b) Probability of handover failure.

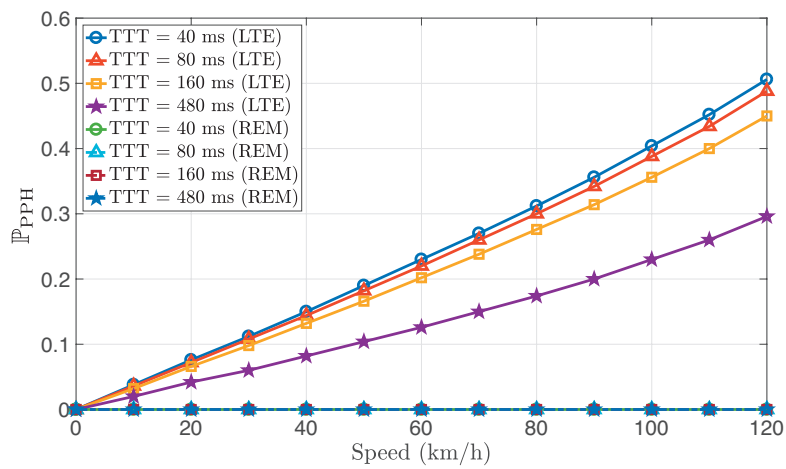

(c) Probability of ping-pong handover.

Fig. 3. Probabilities of not handing over, handover failure and pingpong handover for $\Delta t=2.5 \mathrm{~s}$.

intersect the inbound handover-failure region. Note that not handing over does not imply the call is dropped. Moreover, our proposed algorithm seems to be insensitive to the TTT since the general behavior is the same for all the TTTs considered: a first linear section followed by a saturated one. In Fig. 3 (b), we can observe a similar trend. Above $70 \mathrm{~km} / \mathrm{h}$, the probability of handover failure $\left(\mathbb{P}_{\mathrm{HF}}\right)$ becomes $25.77 \%$. What's happening is that our proposed algorithm avoids all handovers regardless of the speed or the TTT for the considered $\Delta t$, however, the user's trajectories that intersect the out-of-sync region end up having a handover failure. In addition, the LTE-HO algorithm only suffers from handover failures for the longest TTT considered

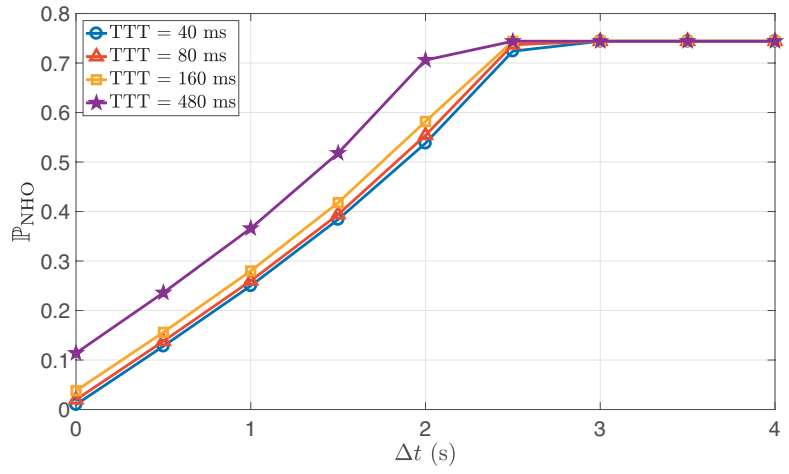

(a) Probability of not handing over.

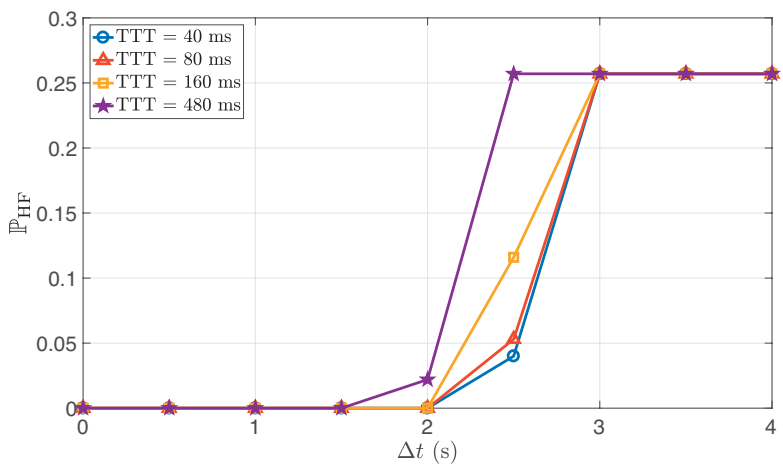

(b) Probability of handover failure.

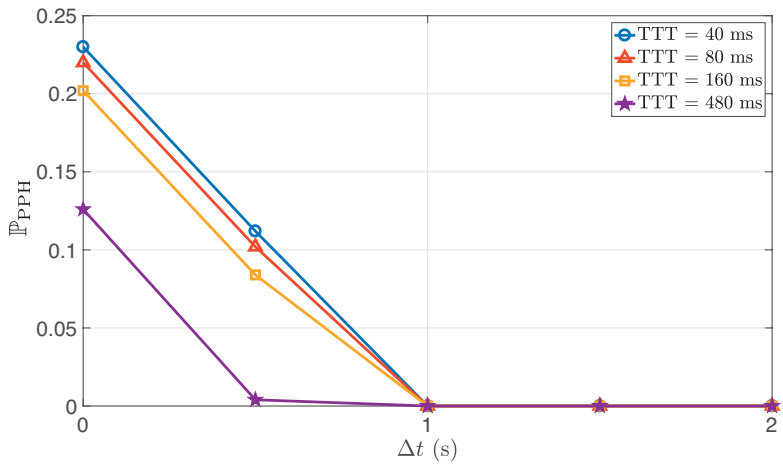

(c) Probability of ping-pong handover.

Fig. 4. Probabilities of not handing over, handover failure and pingpong handover for $60 \mathrm{~km} / \mathrm{h}$.

(480 ms) and high-speed users (above $100 \mathrm{~km} / \mathrm{h}$ ) since, again, the distance $v T$ is longer and the user is more likely to reach the inbound or outbound handoverfailure region. In Fig. 3 (c), the probability of a pingpong handover $\left(\mathbb{P}_{\mathrm{PPH}}\right)$ for our proposed algorithm is non-existent, whereas for the LTE-HO algorithm we can also observe a tradeoff between the probability of not handing over and the ping-pong handover: a longer TTT increases the former and reduces the latter. That is the main reason why most studies presented in Section I try to optimize the TTT.

We are also interested in understanding the relationship between the TTT $T$ and the prediction time $\Delta t$. 
We have selected $60 \mathrm{~km} / \mathrm{h}$, which can be categorized into mid-speed vehicles, and a set of values for $\Delta t$ using the same TTT as in Fig. 3. In Fig. 4 (a), the $\mathbb{P}_{\mathrm{NHO}}$ is basically equivalent to increasing either the TTT or the speed, i.e. the distance. Something similar happens with the $\mathbb{P}_{\mathrm{HF}}$ in Fig. 4 (b), where after a certain point (around $2 \mathrm{~s}$ ) failures start to happen. Here we can see that the prediction time plays an important role to balance the network overhead. Although a longer prediction time avoids handovers, reducing the number of messages between the BSs and the UE, it also produces more handover failures which result in the opposite effect. Finally, the $\mathbb{P}_{\mathrm{PPH}}$ quickly goes to zero for prediction times longer than $1 \mathrm{~s}$ as seen in Fig. 4 (c). Therefore, a design algorithm would need to carefully select the optimal set of parameters for a given network. For example, taking $\Delta t=2 \mathrm{~s}$ we can avoid more than $50 \%$ of the handovers and $100 \%$ of the ping-pong ones while experiencing very little probability of handover failure (below 3\% for $480 \mathrm{~ms}$ ). On the other hand, the LTE counterpart, which is equivalent to $\Delta t=0 \mathrm{~s}$, only avoids $10 \%$ of the handovers (of which between $12 \%$ and $22 \%$ are ping-pong ones). Nonetheless, it does not produce any handover failure.

\section{CONCLUSiON}

In this work, we have presented a novel handover algorithm based on a REM approach that selects the best network access based on the user's course. At the same time, this algorithm avoids performing unnecessary handovers, thus reducing the network overhead on the operator's side as well. Assuming a geometric model, we have also been able to compare the industrystandard algorithm with our proposed one and obtain early findings that can help us to optimize our method. Moreover, we have shown that, for a given prediction time, our method avoids all handovers (100\%) for midand high-speed users with almost $75 \%$ of success $(0 \%$ ping-pong handovers). Nevertheless, it is possible to find a set of feasible points subject to some constraints, e.g. speed, where a reduction of more than $50 \%$ of the handovers is achieved ( $0 \%$ ping-pong handovers) with $97 \%$ of success.

\section{ACKNOWLEDGMENT}

This work has been funded by a Cotutelle International Macquarie University Research Excellence Scholarship (iMQRES) - No. 2016188.

\section{REFERENCES}

[1] "White paper: Cisco VNI Forecast and Methodology, 2016-2021," http://www.cisco.com/c/en/us/solutions/ collateral/service-provider/visual-networking-index-vni/ mobile-white-paper-c11-520862.html, last accessed in May 2017.
[2] $3^{\text {rd }}$ Generation Partnership Project, "Evolved Universal Terrestrial Radio Access (E-UTRA); Mobility enhancements in heterogeneous networks," 3GPP TR 36.839 V11.1.0, Tech. Rep., 2012.

[3] D. Lopez-Perez, I. Guvenc, and X. Chu, "Mobility management challenges in 3GPP heterogeneous networks," IEEE Communications Magazine, vol. 50, no. 12, pp. 70-78, December 2012.

[4] "Spectrum for $4 \mathrm{G}$ and 5G," https://www.qualcomm.com/ documents/spectrum-4g-and-5g, last accessed in May 2017.

[5] D. Xenakis, N. Passas, L. Merakos, and C. Verikoukis, "Mobility Management for Femtocells in LTE-Advanced: Key Aspects and Survey of Handover Decision Algorithms," IEEE Communications Surveys and Tutorials, vol. 16, no. 1, pp. 64-91, 2014.

[6] F. Guidolin, I. Pappalardo, A. Zanella, and M. Zorzi, "ContextAware Handover Policies in HetNets," IEEE Transactions on Wireless Communications, vol. 15, no. 3, pp. 1895-1906, March 2016.

[7] N. P. Kuruvatti, W. Zhou, and H. D. Schotten, "Mobility Prediction of Diurnal Users for Enabling Context Aware Resource Allocation," in 2016 IEEE 83rd Vehicular Technology Conference (VTC Spring), May 2016, pp. 1-5.

[8] X. Yan, Y. A. Sekercioglu, and N. Mani, "A method for minimizing unnecessary handovers in heterogeneous wireless networks," in 2008 International Symposium on a World of Wireless, Mobile and Multimedia Networks, June 2008, pp. 1-5.

[9] H. Zaaraoui, Z. Altman, E. Altman, and T. Jimenez, "Forecast scheduling for mobile users," in 2016 IEEE 27th Annual International Symposium on Personal, Indoor, and Mobile Radio Communications (PIMRC), September 2016, pp. 1-6.

[10] J. Perez-Romero, A. Zalonis, L. Boukhatem, A. Kliks, K. Koutlia, N. Dimitriou, and R. Kurda, "On the use of radio environment maps for interference management in heterogeneous networks," IEEE Communications Magazine, vol. 53, no. 8, pp. 184-191, August 2015

[11] A. Goldsmith, Wireless Communications. New York, NY, USA: Cambridge University Press, 2005.

[12] I. Guvenc, M. R. Jeong, F. Watanabe, and H. Inamura, "A hybrid frequency assignment for femtocells and coverage area analysis for co-channel operation," IEEE Communications Letters, vol. 12 , no. 12 , pp. 880-882, December 2008. 